\title{
REPRESENTATIONS OF THE GUPTA-SIDKI GROUP
}

\author{
D. S. PASSMAN AND W. V. TEMPLE \\ (Communicated by Lance W. Small)
}

\begin{abstract}
If $p$ is an odd prime, then the Gupta-Sidki group $\mathcal{G}_{p}$ is an infinite 2 -generated $p$-group. It is defined in a recursive manner as a particular subgroup of the automorphism group of a regular tree of degree $p$. In this note, we make two observations concerning the irreducible representations of the group algebra $K\left[\mathcal{G}_{p}\right]$ with $K$ an algebraically closed field. First, when char $K \neq p$, we obtain a lower bound for the number of irreducible representations of any finite degree $n$. Second, when char $K=p$, we show that if $K\left[\mathcal{G}_{p}\right]$ has one nonprincipal irreducible representation, then it has infinitely many. The proofs of these two results use similar techniques and eventually depend on the fact that the commutator subgroup $\mathcal{H}_{p}$ of $\mathcal{G}_{p}$ has a normal subgroup of finite index isomorphic to the direct product of $p$ copies of $\mathcal{H}_{p}$.
\end{abstract}

\section{Representations of Finite DEGREE}

If $p$ is an odd prime, then we let $\mathcal{G}_{p}$ denote the Gupta-Sidki group described in [GS]. Specifically, $\mathcal{G}_{p}$ is an infinite 2 -generated $p$-group defined in a recursive manner as a subgroup of the automorphism group of a regular tree of degree $p$. Consequently, $\mathcal{G}_{p}$ is residually finite. In fact, $\mathcal{G}_{p}$ is just infinite [S1] in the sense that every nontrivial normal subgroup of $\mathcal{G}_{p}$ has finite index. For our purposes, a key property of this group is that if $\mathcal{H}_{p}$ is its commutator subgroup, then $\mathcal{H}_{p}$ has a normal subgroup of finite index isomorphic to the direct product of $p$ copies of itself (see [S1]).

If $G$ is any finitely generated group and if all finite-dimensional $K$-representations of $G$ are completely reducible, then a theorem of Weil (see [F]) implies that $G$ has only finitely many $K$-representations of any given finite degree $n$. In particular, this applies to the Gupta-Sidki group $\mathcal{G}_{p}$ provided char $K \neq p$, and motivates the following

Definition. Let $K$ be a fixed algebraically closed field and, for any group $G$, let $f_{G}(n)$ be the number of irreducible representations of $K[G]$ of degree $n$. Furthermore, let $F_{G}(n)=\sum_{i=1}^{n} f_{G}(i)$ be the number of irreducible representations of $K[G]$ of degree $\leq n$. Thus, $f_{G}(n)$ and $F_{G}(n)$ are either nonnegative integers or $\infty$.

Of course, irreducible representations of $K[G]$ correspond in a natural manner to irreducible $K[G]$-modules. We first consider the behavior of the functions $f_{G}$ and $F_{G}$ with respect to normal subgroups of finite index.

Received by the editors November 8, 1994.

1991 Mathematics Subject Classification. Primary 20C07; Secondary 16S34, 20E08, 20F50.

The first author's research supported in part by NSF Grant DMS-9224662. 
Lemma 1.1. Let $H \triangleleft G$ with $|G: H|=k<\infty$ and let $n$ be any positive integer.

(i) $F_{G}(k n) \geq F_{H}(n) / k$ and $F_{H}(n) \geq F_{G}(n) / k$. In particular,

$$
\limsup _{n \rightarrow \infty} \log _{n} F_{G}(n)=\limsup _{n \rightarrow \infty} \log _{n} F_{H}(n) .
$$

(ii) $f_{G}(j n) \geq f_{H}(n) / k^{2}$ for some integer $j=j(n)$ with $1 \leq j \leq k$. Furthermore, if $k>1$ and $f_{H}(n) \neq 0, \infty$, then $f_{G}(j n)>f_{H}(n) / k^{2}$.

Proof. If $V$ is an irreducible $K[H]$-module of degree $t$, then so is $V \otimes g$ for any $g \in G$. In this way, $G / H$ permutes the $F_{H}(n)$ irreducible $K[H]$-modules of degree $\leq n$ and hence there are at least $F_{H}(n) / k$ orbits under this action. Notice that the restriction of the induced $K[G]$-module $V^{G}=V \otimes_{K[H]} K[G]$ to $K[H]$ is a direct sum of $k$ modules of the form $V \otimes g$. Thus, if $X$ is any irreducible $K[G]$ constituent of $V^{G}$, then $X_{H}$ is isomorphic to a direct sum of appropriate $V \otimes g$. Hence $\operatorname{deg} X=j \operatorname{deg} V \leq j n$ and $X$ determines the orbit of $V$. In other words, each $G$-orbit of $K[H]$-modules of degree $\leq n$ gives rise to at least one irreducible $K[G]$-module of degree $\leq k n$ and hence $F_{G}(k n) \geq F_{H}(n) / k$.

Similarly, there are at least $f_{H}(n) / k$ orbits of irreducible $K[H]$-modules of degree $n$, and therefore the above implies that

$$
f_{G}(n)+f_{G}(2 n)+\cdots+f_{G}(k n) \geq f_{H}(n) / k .
$$

In particular, if $f_{G}(j n)$ is the largest of the left-hand summands, then $k f_{G}(j n) \geq$ $f_{H}(n) / k$ and this is the required inequality for (ii). Note that if equality occurs here, then we must have precisely $f_{H}(n) / k$ orbits under the action of $G / H$, and therefore $G / H$ acts semiregularly. But this implies that if $V$ if an irreducible $K[H]$-module of degree $n$, then the induced module $V^{G}$ is also irreducible. Thus, we obtain at least $f_{H}(n) / k$ irreducible $K[G]$-modules of degree $n k$, so $f_{H}(n) / k^{2}=f_{G}(j n) \geq$ $f_{G}(k n) \geq f_{H}(n) / k$ and hence if $k>1$, then $f_{H}(n)=0$ or $\infty$.

Finally, for the reverse inequality in (i), let $W$ be an irreducible $K[G]$-module of degree $\leq n$, and let $V$ be any irreducible constituent of $W_{H}$. Then $\operatorname{deg} V \leq n$ and $W$ is a composition factor of $V^{G}$. Since there are at most $k$ composition factors of $V^{G}$, it follows that there are at most $k$ such $W$ which give rise to $V$, and therefore $F_{H}(n) \geq F_{G}(n) / k$. In particular, $\log _{n} F_{H}(n) \geq \log _{n} F_{G}(n)-\log _{n} k$, so

$$
\limsup _{n \rightarrow \infty} \log _{n} F_{H}(n) \geq \limsup _{n \rightarrow \infty} \log _{n} F_{G}(n)
$$

since $\lim _{n} \log _{n} k=0$. Furthermore, the first inequality in (i) yields

$$
\log _{k n} F_{G}(k n)=\frac{\log _{n} F_{G}(k n)}{1+\log _{n} k} \geq \frac{\log _{n} F_{H}(n)-\log _{n} k}{1+\log _{n} k},
$$

so

$$
\limsup _{n \rightarrow \infty} \log _{n} F_{G}(n) \geq \limsup _{n \rightarrow \infty} \log _{k n} F_{G}(k n) \geq \limsup _{n \rightarrow \infty} \log _{n} F_{H}(n)
$$

and the lemma is proved.

Next we consider a special case which is obviously of interest here. 
Lemma 1.2. Let $G$ have a normal subgroup $H$ of finite index $k$ and suppose that $H \cong G \times G \times \cdots \times G$, the direct product of $q$ copies of $G$.

(i) $F_{G}\left(k n^{q}\right) \geq F_{G}(n)^{q} / k$ for all $n$. In particular, if $F_{G}(1) \geq k>1$, then the inequality $F_{G}(n) \geq k n^{q-2}$ is satisfied for infinitely many $n$ and hence

$$
\limsup _{n \rightarrow \infty} \log _{n} F_{G}(n) \geq q-2 .
$$

(ii) $f_{G}\left(j n^{q}\right) \geq f_{G}(n)^{q} / k^{2}$ for all $n$, where $j=j(n)$ satisfies $1 \leq j \leq k$. Furthermore, if $k>1$ and $f_{G}(n) \neq 0, \infty$, then $f_{G}\left(j n^{q}\right)>f_{G}(n)^{q} / k^{2}$. In particular, if $k>1$ and $f_{G}(n) \geq k^{2 /(q-1)}$ for some $n$, then $f_{G}$ is an unbounded function.

Proof. If $V_{1}, V_{2}, \ldots, V_{q}$ are irreducible $K[G]$-modules of finite degree, then, since $K$ is algebraically closed, it follows that $V=V_{1} \otimes V_{2} \otimes \cdots \otimes V_{q}$ is an irreducible $K[H]$ module with $\operatorname{deg} V=\prod_{i} \operatorname{deg} V_{i}$. Furthermore, all such $V$ obtained in this manner are distinct. Thus, for any integer $n$, we have $F_{H}\left(n^{q}\right) \geq F_{G}(n)^{q}$ and Lemma 1.1(i) implies that

$$
F_{G}\left(k n^{q}\right) \geq F_{H}\left(n^{q}\right) / k \geq F_{G}(n)^{q} / k .
$$

Now assume that $k>1$ and that $F_{G}(n) \geq k n^{q-2}$. Then $m=k n^{q}>n$ and

$$
F_{G}(m)=F_{G}\left(k n^{q}\right) \geq F_{G}(n)^{q} / k \geq\left(k n^{q-2}\right)^{q} / k=k m^{q-2} .
$$

Thus, if $F_{G}(1) \geq k>1$, then it follows from the above that the inequality $F_{G}(n) \geq$ $k n^{q-2}$ is satisfied for $n=1$ and hence for infinitely many $n$. Consequently,

$$
\limsup _{n \rightarrow \infty} \log _{n} F_{G}(n) \geq \lim _{n \rightarrow \infty} \log _{n} k n^{q-2}=q-2 .
$$

Similarly, for all $n \geq 1$, we have $f_{H}\left(n^{q}\right) \geq f_{G}(n)^{q}$ and hence, by Lemma 1.1(ii),

$$
f_{G}\left(j n^{q}\right) \geq f_{H}\left(n^{q}\right) / k^{2} \geq f_{G}(n)^{q} / k^{2}
$$

for some $1 \leq j \leq k$. Furthermore, if $k>1$ and $f_{G}(n) \neq 0, \infty$, then the preceding inequality is strict. Finally, suppose that $k>1$ and that $f_{G}$ is a bounded function with the maximum value equal to $f_{G}(m) \neq 0$. Then

$$
f_{G}(m) \geq f_{G}\left(j m^{q}\right)>f_{G}(m)^{q} / k^{2}
$$

and $k^{2 /(q-1)}>f_{G}(m)$. In particular, if $f_{G}(n) \geq k^{2 /(q-1)}$ for some $n$, then $f_{G}$ must be an unbounded function.

As we mentioned earlier, the structure of the Gupta-Sidki group is similar to that of the group in the preceding lemma. Consequently, we obtain

Theorem 1.3. Let $p$ be an odd prime and let $\mathcal{G}_{p}$ be the Gupta-Sidki group corresponding to $p$. If $K$ is an algebraically closed field of characteristic $\neq p$ and if $F(n)$ denotes the number of irreducible representations of $K\left[\mathcal{G}_{p}\right]$ of degree $\leq n$, then $F$ is a finite-valued function and

$$
\limsup _{n \rightarrow \infty} \log _{n} F(n) \geq p-2 .
$$


In particular, if $f(n)$ denotes the number of irreducible representations of $K\left[\mathcal{G}_{p}\right]$ of degree precisely $n$, then the function $f$ is finite valued and unbounded.

Proof. Both $F$ and $f$ are finite valued by Weil's theorem. For convenience, write $G=\mathcal{G}_{p}$ and let $H=G^{\prime}$ be the commutator subgroup of $\mathcal{G}_{p}$. Then $H \triangleleft G$ and $|G / H|<\infty$, so

$$
\limsup _{n \rightarrow \infty} \log _{n} F_{G}(n)=\limsup _{n \rightarrow \infty} \log _{n} F_{H}(n)
$$

by Lemma 1.1(i). Next, $[\mathrm{S} 1, \S 2]$ implies that $H$ has a normal subgroup $L$ of finite index $k>1$ with $L \cong H \times H \times \cdots \times H$, the direct product of $p$ copies of $H$. Furthermore, $H / L$ is an abelian $p$-group, so $K[H / L]$ has $k$ irreducible linear representations and hence $F_{H}(1) \geq k$. Thus, by Lemma 1.2(i),

$$
\limsup _{n \rightarrow \infty} \log _{n} F_{G}(n)=\limsup _{n \rightarrow \infty} \log _{n} F_{H}(n) \geq p-2
$$

as required.

Finally, if $f_{G}$ were a bounded function, then $F_{G}(n) \leq b n$ for some bound $b$ and hence $\lim \sup _{n} \log _{n} F_{G}(n) \leq 1$. Thus the result about $f_{G}$ follows directly from the above estimate if $p \geq 5$. On the other hand, a proof for all $p \geq 3$ can be obtained easily from the second parts of the preceding two lemmas. Indeed, since $f_{H}(1) \geq k \geq k^{2 /(p-1)}$, Lemma 1.2(ii) implies that $f_{H}$ is an unbounded function, and then Lemma 1.1(ii) implies the same for $f_{G}$.

We remark that the original motivation for the preceding result came from the paper $[\mathrm{KS}]$ which studied algebras with representation properties analogous to those of $K\left[\mathcal{G}_{p}\right]$. For example, [KS] observed that certain enveloping algebras $U(L)$ satisfied the conclusion of Weil's theorem in the stronger sense that they had a uniformly bounded finite number of irreducible representations of any given finite degree. Thus, we wondered whether this stronger property might also hold for $K\left[\mathcal{G}_{p}\right]$, but it does not since the function $f$ is unbounded. Of course, it would still be interesting to obtain the exact value of the parameter $\lim \sup _{n} \log _{n} F(n)$ since this number is

a measure of the growth of the collection of finite-degree irreducible representations of the group algebra.

\section{Modular REPRESEntations}

Again, let $\mathcal{G}_{p}$ denote the Gupta-Sidki group associated with the prime $p$, but now let $K$ be a field of characteristic $p$. Then $K\left[\mathcal{G}_{p}\right]$ is a modular group algebra which we nevertheless believe to be semiprimitive. But until recently, no examples of nonprincipal irreducible representations for this algebra were known to exist. Fortunately, paper [S2] has now shown that if $p=3$, then $K\left[\mathcal{G}_{3}\right]$ has an infinitedimensional primitive homomorphic image.

In this section, we prove that if $K\left[\mathcal{G}_{p}\right]$ has one nonprincipal irreducible representation, then it has infinitely many. More precisely, we do this under the additional assumption that $K$ is algebraically closed and nondenumerable. In the following, we fix a field $K$ and we use $\# G$ to denote the number of irreducible representations of the group algebra $K[G]$. Thus $\# G$ is either a nonnegative integer or $\infty$. As usual, we start with 
Lemma 2.1. Let $H \triangleleft G$ with $|G: H|=k<\infty$. Then, for any field $K$, we have $\# G \geq \# H / k$ and $\# H \geq \# G / k$.

Proof. We know that $G / H$ permutes the set of irreducible $K[H]$-modules and that there are at least $\# H / k$ orbits under this action. Thus, since each such orbit gives rise to at least one irreducible $K[G]$-module, it follows that $\# G \geq \# H / k$.

Conversely, let $W$ be an irreducible $K[G]$-module and observe that $W$ is a homomorphic image of $\left(W_{H}\right)^{G}$. Consequently, if $V$ is an irreducible constituent of $W_{H}$, then $W$ is a constituent of $V^{G}$. But $V^{G}$ has at most $k$ composition factors, so there are at most $k$ such $W$ which give rise to any particular $V$. Thus we obtain at least $\# G / k$ irreducible $K[H]$-modules in this manner, and therefore $\# H \geq \# G / k$.

As expected, our proof that $\# \mathcal{G}_{p}$ is infinite depends on the fact that $\mathcal{G}_{p}^{\prime}$ has a normal subgroup of finite index isomorphic to a direct product of $p$ copies of itself. But here we require a more precise understanding of the inclusion and therefore it is best to skip any additional lemmas of a general nature. In other words, we proceed directly to

Theorem 2.2. Let $p$ be an odd prime, let $\mathcal{G}_{p}$ be the Gupta-Sidki group corresponding to $p$, and let $K$ be a nondenumerable algebraically closed field of characteristic $p>0$. If the modular group algebra $K\left[\mathcal{G}_{p}\right]$ has a nonprincipal irreducible representation, then $K\left[\mathcal{G}_{p}\right]$ has infinitely many irreducible representations.

Proof. For convenience, let $G=\mathcal{G}_{p}$ and $H=\mathcal{G}_{p}^{\prime}$. Furthermore, let $L$ be the normal subgroup of $H$ of finite index with $L \cong H \times H \times \cdots \times H$, the direct product of $p$ copies of $H$. If $V$ is an irreducible $K[H]$-module and if $D=\operatorname{End}_{K[H]} V$ is its commuting ring, then $D$ is a countable-dimensional division algebra over $K$. Thus, since $K$ is nondenumerable and algebraically closed, it follows that $D=K$. As a consequence, if $V_{1}, V_{2}, \ldots, V_{p}$ are irreducible $K[H]$-modules, then $V_{1} \otimes V_{2} \otimes \cdots \otimes V_{p}$ is an irreducible $K[L]$-module. Set $n=\# H$ and let $K_{0}$ denote the principal module for both $K[G]$ and $K[H]$.

We first reduce considerations to $K[H]$. To this end, suppose $U$ is the given nonprincipal irreducible $K[G]$-module and observe that $U_{H}=U_{1}^{\prime} \oplus U_{2}^{\prime} \oplus \cdots \oplus U_{t}^{\prime}$ is a finite direct sum of irreducible $K[H]$-modules. If all $U_{i}^{\prime} \cong K_{0}$, then $U$ is an irreducible $K[G / H]$-module. But $G / H$ is a finite $p$-group and char $K=p$, so this implies that $U \cong K_{0}$, a contradiction. In other words, we have shown that $K[H]$ has a nonprincipal irreducible module and, in view of Lemma 2.1, it suffices to show that $n=\# H$ is infinite.

Suppose, by way of contradiction, that $n$ is finite. We will obtain two opposing estimates for the size of $n$. To start with, if $H$ and $L$ are as above, then [S1,§2] implies that $H / L$ is abelian of order $k \leq p^{2 p}$. Furthermore, by taking tensor products of all $K[H]$-modules, we see that $K[L]$ has at least $n^{p}$ distinct irreducible modules. Thus, by Lemma 2.1,

$$
n=\# H \geq \# L / k \geq n^{p} / k,
$$

so $p^{2 p} \geq k \geq n^{p-1} \geq n^{2}$ and hence $p^{p} \geq n$.

To obtain a lower bound for $n$, we again note that

$$
G \supseteq H \supseteq L=L_{1} \times L_{2} \times \cdots \times L_{p}
$$


and that $L \triangleleft G$ and $L_{i} \cong H$. Furthermore, each factor $L_{i}$ of $L$ is normal in $H$, and $G$ permutes these factors by conjugation. Let $X$ be the given nonprincipal irreducible $K[H]$-module, and view it as a module for each $K\left[L_{i}\right]$. Then, we can consider the $p+1$ irreducible $K[L]$-modules defined by

$$
Y_{j}=\underbrace{X \otimes X \otimes \cdots \otimes X}_{j \text { times }} \otimes \underbrace{K_{0} \otimes K_{0} \otimes \cdots \otimes K_{0}}_{p-j \text { times }}
$$

for $j=0,1, \ldots, p$. Since $X \neq K_{0}$ and $G$ permutes the factors $L_{i}$ of $L$, it follows that $Y_{0}, Y_{1}, \ldots, Y_{p}$ are in distinct orbits under the action of $G$.

For each $j$, let $Z_{j}$ be an irreducible constituent of $Y_{j}^{H}$. Since the $Y_{j}$ are in distinct $H$-orbits, it follows that $Z_{0}, Z_{1}, \ldots, Z_{p}$ are distinct irreducible $K[H]$-modules. In fact, since $Y_{j}$ is a direct summand of $\left(Z_{j}\right)_{L}$, it follows that $Z_{0}, Z_{1}, \ldots, Z_{p}$ are in distinct orbits under the action of $G$ on the irreducible $K[H]$-modules. Again, we view these $Z_{j}$ as $K\left[L_{i}\right]$-modules and we consider the $(p+1)^{p}$ distinct irreducible $K[L]$-modules of the form $Z_{j_{1}} \otimes Z_{j_{2}} \otimes \cdots \otimes Z_{j_{p}}$. From the structure of $G$ as described in $[\mathrm{S} 1, \S 2]$, we see that $H$ normalizes each $L_{i}$ and acts on each $L_{i} \cong H$ as $G$ acts on $H$. Thus, since the $Z_{j}$ are in distinct $G$-orbits, it follows that these $(p+1)^{p}$ tensor product modules are in distinct $H$-orbits. In particular, they give rise to at least $(p+1)^{p}$ distinct irreducible $K[H]$-modules. In other words, we have shown that $n \geq(p+1)^{p}$ and, since this contradicts the previous upper bound estimate $n \leq p^{p}$, the result follows.

As an immediate consequence of the above and the main theorem of [S2], we obtain

Corollary 2.3. Let $\mathcal{G}_{3}$ be the Gupta-Sidki group corresponding to the prime 3 , and let $K$ be a nondenumerable algebraically closed field of characteristic 3 . Then the modular group algebra $K\left[\mathcal{G}_{3}\right]$ has infinitely many irreducible representations.

Hopefully, the work of [S2] is just a first step toward proving the semiprimitivity of $K\left[\mathcal{G}_{p}\right]$ for all odd primes $p$.

\section{Affine algebras}

Let $K$ be any field and recall that an affine $K$-algebra is just a finitely generated $K$-algebra. Thus, for example, if $G$ is a finitely generated group, then the group algebra $K[G]$ is affine. Indeed, as was observed in [F], Weil's theorem is really a result about affine $K$-algebras. In this final section, we offer a few brief comments on algebras of this type and we start with a standard fact.

Lemma 3.1. Let $A$ be an affine $K$-algebra.

(i) If $V$ is a finitely generated $A$-module and if $W$ is a submodule of finite codimension, then $W$ is also finitely generated.

(ii) Every right ideal of $A$ of finite codimension is finitely generated. In particular, if $I$ and $J$ are ideals of $A$ of finite codimension, then so is $I J$.

Proof. (i) Let $A$ be generated by $a_{1}, a_{2}, \ldots, a_{n}$ and choose a $K$-subspace $X \subseteq$ $V$ which complements $W$. By assumption, $X$ is finite dimensional with $K$-basis $x_{1}, x_{2}, \ldots, x_{m}$, and let $v_{1}, v_{2}, \ldots, v_{t}$ generate $V$ as an $A$-module. Since $V=W \oplus X$, there is a natural projection map $\pi: V \rightarrow W$, and we let $W_{0}$ be the $A$-submodule of $W$ generated by the finitely many elements $\pi\left(x_{i} a_{j}\right)$ and $\pi\left(v_{k}\right)$ for all $i, j, k$. 
Since $v \in \pi(v) \oplus X$ for any $v \in V$, it follows that $x_{i} a_{j}, v_{k} \in W_{0} \oplus X$ for all $i, j, k$. With this, it is easy to see that $W_{0} \oplus X$ is a submodule of $V$ containing the generators $v_{1}, v_{2}, \ldots, v_{t}$. In other words, $W_{0} \oplus X=V=W \oplus X$ and, since $W_{0} \subseteq W$, it follows that $W=W_{0}$ is finitely generated.

(ii) Since $A$ is a finitely generated right $A$-module, part (i) above implies that any right ideal of $A$ of finite codimension is finitely generated. Now let $I$ and $J$ be ideals of $A$ of finite codimension. Then $I / I J$ is a finitely generated right $A / J$-module, so $\operatorname{dim}_{K} I / I J<\infty$. Thus, since $\operatorname{dim}_{K} A / I<\infty$, the result follows.

As a consequence, we obtain a lovely observation of L. W. Small. Recall that a $K$-algebra $A$ is said to be just infinite if $A$ is infinite dimensional, but all proper homomorphic images of $A$ are finite dimensional.

Proposition 3.2. Let $A$ be an infinite-dimensional affine $K$-algebra. Then $A$ has a homomorphic image $\bar{A}$ which is prime and just infinite.

Proof. Since all ideals of $A$ of finite codimension are finitely generated, it follows from Zorn's lemma that $A$ has an ideal $M$ maximal with the property that $\operatorname{dim}_{K} A / M$ is infinite. Thus $\bar{A}=A / M$ is a just-infinite homomorphic image of $A$ and, of course, $\bar{A}$ is also affine. Finally, if $\bar{I}$ and $\bar{J}$ are nonzero ideals of $\bar{A}$, then they have finite codimension, so Lemma 3.1(ii) implies that $\bar{I} \bar{J}$ also has finite codimension. In particular, $\bar{I} \bar{J} \neq 0$ and $\bar{A}$ is prime.

It follows from the above that if $G$ is any finitely generated infinite group, then $K[G]$ has a prime ideal $P$ of infinite codimension. Of course, this can also be proved directly from the general description of the prime radical of $K[G]$ as given in [DZ] or in [P, Theorem 8.4.16]. We remark that the infinite-dimensional primitive homomorphic image of $K\left[\mathcal{G}_{3}\right]$ constructed in [S2] is a prime, just-infinite $K$-algebra. Thus, the real content of the main result of [S2] is that such an image can be chosen to be primitive.

Finally, we can use the preceding result to give slightly shorter and more transparent proofs of the algebraic generalizations of Weil's theorem. For example, the following is [BG, Proposition 2.1].

Theorem 3.3. Let $A$ be an affine $K$-algebra satisfying a polynomial identity, and let $d$ be a fixed integer. If the nil radical of every finite-dimensional homomorphic image of $A$ has nilpotence degree at most $d$, then A itself is finite dimensional.

Proof. Suppose, by way of contradiction, that $\operatorname{dim}_{K} A=\infty$. Since the hypotheses on $A$ are inherited by homomorphic images, Proposition 3.2 allows us to assume that $A$ is prime. Hence, since $A$ satisfies a polynomial identity, Posner's theorem (see [H, Theorem 7.3.2]) implies that $A$ is contained in $\mathrm{M}_{n}(F)$, the ring of $n \times n$ matrices over some field extension $F$ of $K$. Indeed, since $A$ is a finitely generated $K$-algebra, we can assume that $A \subseteq \mathrm{M}_{n}(R)=\mathcal{R}$, where $R$ is a commutative affine $K$-algebra contained in $F$. Let $I$ be a maximal ideal of $R$ and set $\mathcal{I}=\mathrm{M}_{n}(I) \triangleleft \mathcal{R}$.

By the Hilbert Nullstellensatz, $I$ has finite codimension in $R$ and hence, by Lemma 3.1(ii), the same is true of any $I^{k}$. In particular, $\mathcal{R} / \mathcal{I}^{k}$ is a finite-dimensional homomorphic image of $\mathcal{R}$ containing $\bar{A}=A /\left(A \cap \mathcal{I}^{k}\right)$. But $(A \cap \mathcal{I}) /\left(A \cap \mathcal{I}^{k}\right)$ is a nilpotent ideal of $\bar{A}$ so, by assumption,

$$
(A \cap \mathcal{I})^{d} \subseteq A \cap \mathcal{I}^{k} \subseteq \mathcal{I}^{k}=\mathrm{M}_{n}\left(I^{k}\right) .
$$


Thus, since $\bigcap_{k=1}^{\infty} I^{k}=0$ by the Krull Intersection Theorem, we have $(A \cap \mathcal{I})^{d}=0$. Finally, $A \cap \mathcal{I}$ has finite codimension in the affine algebra $A$, so the same is true of $(A \cap \mathcal{I})^{d}$ by Lemma 3.1(ii). Hence $\operatorname{dim}_{K} A=\operatorname{dim}_{K} A /(A \cap \mathcal{I})^{d}<\infty$ and the theorem is proved.

Of course, when $d=0$, this is the main result of $[\mathrm{F}]$. A short geometric proof of the latter also appears in $[\mathrm{KS}]$.

\section{REFERENCES}

[BG] A. D. Bell and K. R. Goodearl, Algebras of bounded finite dimensional representation type, Glasgow Math. J. (to appear).

[DZ] Z.Z. Dyment and A. E. Zalesskiǔ, On the lower radical of a group ring, in Russian, Dokl. Akad. Nauk BSSR 19 (1975), 876-879. MR 52:5723

[F] D. R. Farkas, Semisimple representations and affine rings, Proc. Amer. Math. Soc. 101 (1987), 237-238. MR 88h:16027

[GS] N. Gupta and S. Sidki, On the Burnside problem for periodic groups, Math. Z. 182 (1983), 385-388. MR 85g:20075

[H] I. N. Herstein, Noncommutative Rings, Carus Math. Monograph \#15, M.A.A., 1968. MR 37:2790

[KS] H. Kraft and L. W. Small, Invariant algebras and completely reducible representations, Math. Research Letters 1 (1994), 297-307. CMP 95:04

[P] D. S. Passman, The Algebraic Structure of Group Rings, Wiley-Interscience, New York, 1977. MR 81d: 16001

[S1] S. Sidki, On a 2-generated infinite 3-group: subgroups and automorphisms, J. Algebra 110 (1987), 24-55. MR 89b:20081b

[S2] _ A primitive ring associated to a Burnside 3-group (to appear).

Department of Mathematics, University of Wisconsin, Madison, Wisconsin 53706

Current address, W. V. Temple: ExperTune Software, 4734 Sonseeahray Drive, Hubertus, Wisconsin 53033 